\title{
Control of surface subsidence based on building deformation monitoring data
}

\author{
Igor Shardakov ${ }^{1 *}$, Aleksandr Barayakh $^{2}$, Valery Yepin ${ }^{1}$, Roman Tsvetkov $^{1}$, and Irina Glot ${ }^{1}$ \\ ${ }^{1}$ Institute of Continuous Media Mechanics, UB RAS, 614013, Perm, Russia \\ ${ }^{2}$ Mining Institute UB RAS, 614990, Perm, Russia
}

\begin{abstract}
This paper presents an approach to the estimation of ground surface distortion based on the data from the online deformation monitoring systems mounted on the foundations of the group of buildings located in the area of ground instability. The local monitoring systems provide control of building foundation settlements using the hydrostatic level measurement technique. These data are used to calculate the inclination foundation angles which reflect the distortion of the earth's surface at local points. The hydrostatic level system allows one to perform measurements with the desired space and time sampling and to obtain a quite detailed picture of the changes in the deformation parameters over time. A set of such local monitoring units forms a distributed system that allows monitoring the state of the earth's surface over a large area. Here, we present long-term results obtained using such system located in the city area above mining. The evolution of the inclination angles of the group of overlying buildings is shown. We discuss the validity of this approach, and estimate the accuracy of the measuring method and the factors that influence it. Finally, we assess the possibility of making short-term predictions of deformation processes inside the rock massif.
\end{abstract}

\section{Introduction}

The deformation processes occurring in rock formations can create hazard to overlying engineering structures and buildings. The processes can be provoked by mining and get worse in an emergency condition. The processes occurring inside rocks are of rather complicated nature, from deformations of rocks to the generation of damages and fractures, surface subsidence and its manifestation on the surface - sinkholes. There is a problem, both for mathematical modelling of this process and for its experimental registration, associated with large scales of the research area as well as with a lack of information about the processes occurring inside the massif.

One way to control the processes running in the earth interior and prevent their detrimental effects is monitoring of various geomechanical parameters such as subsidence of the Earth's surface, which responds to the processes initiated inside the rock massif. There are different approaches to controlling this surface, from instrumental observations [1] to laser-scanning technologies [2,3] and satellite technologies. These methods have

* Corresponding author: shardakov@icmm.ru 
their own benefits and drawbacks. For example, satellite interferometry SAR [4] can control large areas of the surface, but it is impossible to organize these measurements with sufficient frequency (the possible periodicity of these measurements is several weeks). Moreover, there are restrictions on the application of this technology associated with the certain states of the earth's surface (snow cover, vegetation, etc.). The position of individual points on the earth's surface can be monitored in real time using instrumental observations and also with the use of GPS receivers [5-7]. Control of the shape of the earth's surface with GPS receivers with good resolution on a large spatial scale has its own problems, such as high measurement costs. In addition, this requires that the space between the satellites and the surface being surveyed should be free of foreign objects [8].

In urban construction areas, continuous control of the ground surface subsidence can be implemented by means of distributed monitoring systems, which are based on control of the deformation states of individual structures. This approach was proposed in our earlier work [9], where the data on the vertical displacements of the foundations of a set of buildings located above the mine working were used to estimate changes in the tilt angles of the ground surface over a sufficiently wide area. It was assumed that the buildings incline as a unit rigid body. In this paper, we discuss the validity of this approach, and estimate the accuracy of the measuring method and the factors that influence it.

\section{Estimation of the tilt of the earth's massif surface based on the data of monitoring the individual buildings}

In work [10], the authors give an estimate of the geometric dimensions of the deformed earth's surface, the significant distortions of which are due to underlying mine workings. The section of this surface cut by a vertical plane (Fig. 1) is called the subsidence profile and is illustrative of geometrical parameters, characterizing the scales and the shape of the distorted surface.

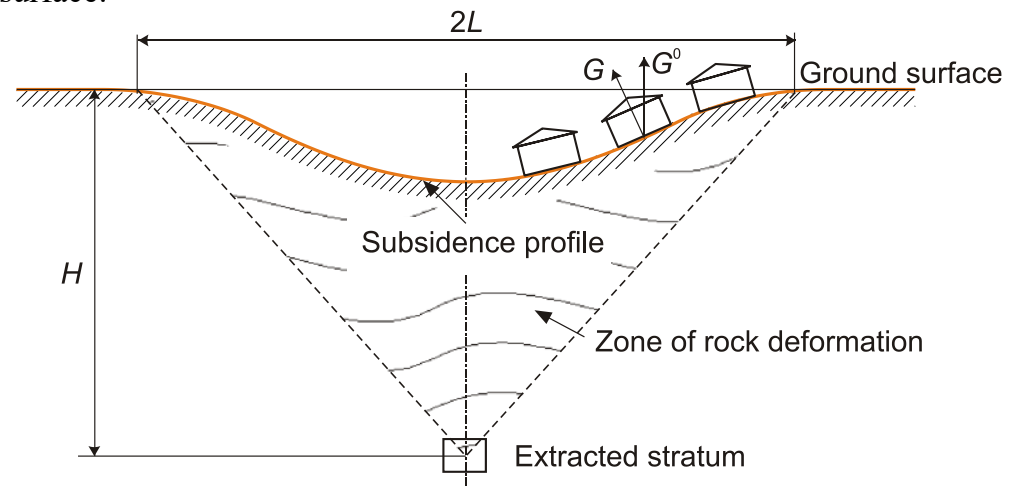

Fig. 1. The scheme of changes of the earth's surface overlying the mine working

The characteristic size $2 \mathrm{~L}$ of the deformed part of the surface is determined by the bedding depth $H$. The ratio $L / H$ depends on the size of the mine working and the mechanical properties of the rock strata. For example, in [10] it is noted that for coal mines this ratio is 0.7 and, for salt deposits, it is in the range from 1.65 to 1.85 [11]. For salt deposits, exploited at depths $H=300 \div 400 \mathrm{~m}$, according to estimates $[12,13]$, the distance $L$ is $500 \div 600 \mathrm{~m}$. The presented estimates of the characteristic sizes and shapes of the deformed part of the earth's surface allow us to conclude that it is possible to estimate such distortions of the surface, using the information obtained from the objects of a smaller scale located on it. So, if we imagine that an ordinary house, which has a characteristic size much smaller than $L$, is an absolutely rigid body connected to the surface, then this house can be 
considered a kind of sensor that reflects the state of the normal to the earth's surface at a local point of a large spatial region. Having information on the change in the angle of inclination of a group of such sensor buildings, it is possible to calculate by means of interpolation a continuous field of inclination angles and to return the shape of the distorted surface. Thus, it is possible to estimate the distortions of the earth's surface on a sufficiently large scale (for example, in the territory of a city district). Information about the long-term behavior of these sensor buildings makes it possible to monitor the evolution of the earth's surface within the observed region. This information is extremely important for the prediction of deformation processes in the rock massif.

To measure the inclination of individual building, we use the data obtained when recording the relative settlements of the characteristic points of its basement. We have developed an automated system of deformation monitoring of the individual building, based on the method of hydrostatic leveling [14]. The measurement of the difference in vertical displacements makes it possible to evaluate the inhomogeneous deformations of the basement, and also to observe the change in the position of the foundation as a rigid body.

The method of determining the inclination of the foundation of a building as a unit rigid body based on the data of vertical displacement sensors of the hydrostatic level system (HLS) is as follows. At the beginning of monitoring, it is assumed that the foundation is a plane S, the unit normal of which $G_{0}$ coincides with the vertical $z$-axis (Fig. 2). During monitoring, the sensors record vertical displacements $U_{i}(i=1 . . N)$ at $N$ points with coordinates $\left(x_{i}, y_{i}\right)$ located on the building foundation. A set of discrete values of these displacements makes it possible to construct a surface $U$ corresponding to the deformed state of the foundation at each instant of time. From this deformation state, one can single out a component responsible for a rigid-body rotation of the foundation relative to its initial position. To this end, it is necessary to construct a plane $S^{*}(x, y)=a x+b y+c$, whose coefficients $(a, b, c)$ are determined from the condition of minimum quadratic deviation $\min _{a, b, c}\left[\sum_{i=1}^{N}\left(U\left(x_{i}, y_{i}\right)-S^{*}\left(x_{i}, y_{i}\right)\right)^{2}\right]$.

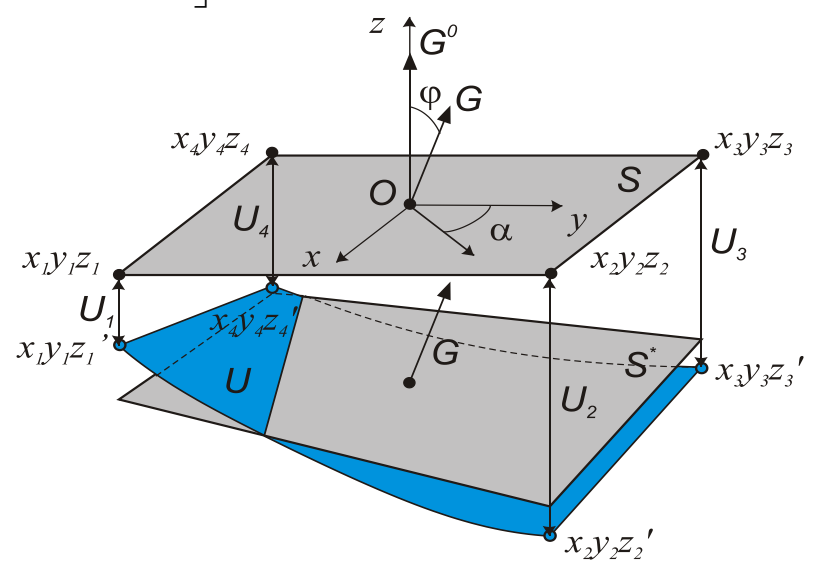

Fig. 2. Illustration of the method of estimating the inclination angle using foundation settlement monitoring data

The position of the unit normal $G$ at any time with respect to the coordinate system $(x, y, z)$ is defined by two quantities: the angle $\varphi$ between the $z$-axis and the unit normal, and the angle $\alpha$ between the $x$-axis and the normal projection onto the $x O y$ plane. The first angle characterizes the slope of the normal, and the second - its direction. Assuming that the foundation is rigidly fixed to the ground surface, we can suppose that these parameters 
determine a change in the angle of inclination of the normal to the ground surface on the scale of the building.

\section{The results of individual building deformation monitoring}

In the developed monitoring system for an individual building, the following values are used to estimate the deformation state of building foundation:

- differential settlement $\delta S_{i, j}=U_{i}-U_{j}$ is equal to the difference of vertical displacements of two foundation points $i$ and $j$ where sensors are located;

- angular distortion is estimated from vertical displacements of two sensors $i$ and $j$ having distance $L_{i, j}: \beta=\frac{\delta S_{i, j}}{L_{i, j}}$

- the curvature of the foundation between sensors $i-1, i, i+1$ is estimated from three reference points where vertical displacements are recorded:

$$
k=\frac{2 \cdot \delta S_{i-1}}{L_{i-1, i} \cdot L_{i+1, i-1}}-\frac{2 \cdot \delta S_{i}}{L_{i-1, i} \cdot L_{i, i+1}}+\frac{2 \cdot \delta S_{i+1}}{L_{i+1, i-1} \cdot L_{i, i+1}} .
$$

Data on vertical displacements of the characteristic points of building foundation, supplied by the monitoring system, can be used to assess the stress-strain state of the building at a time of observation and to analyze its changes over time. In addition, based on the finite element modelling it is possible to estimate the change in the stress-strain state of building elements, using the measured values of vertical displacements at the sensor location points. For this purpose, the analytical part of the monitoring system has a block of finite element simulation, which uses the information on current values of vertical displacements at measuring points as boundary conditions for solving the problem of evaluation the stressstrain state of the building. Such local deformation monitoring systems were developed and installed to control the deformation behaviour of 40 buildings located in the urban area above the salt mines.

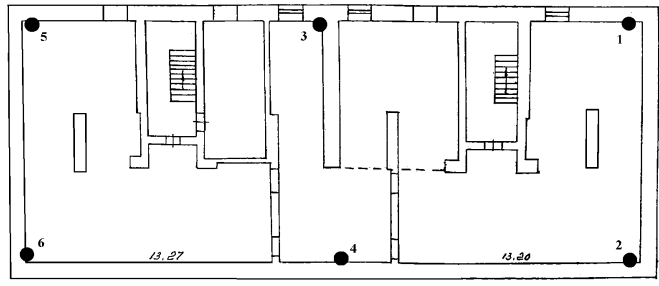

a

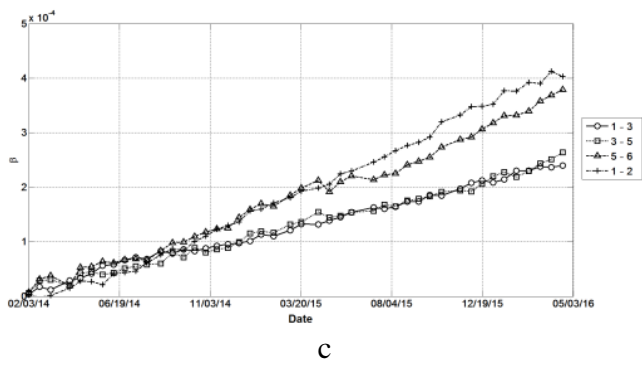

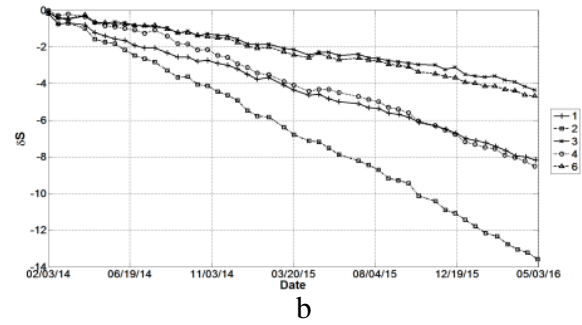

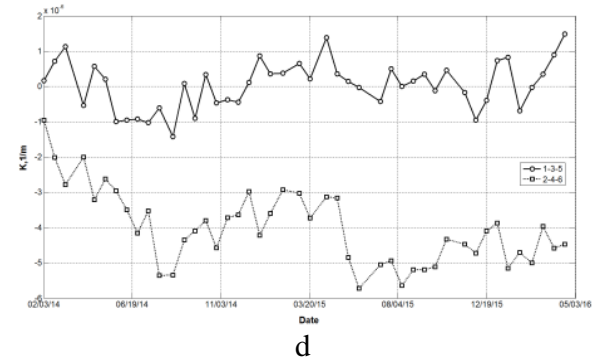

Fig. 3. (a) Sensor layout; (b) vertical displacement at sensor location points; (c) angular distortion of the part of foundation between 2 sensors; (d) curvature of the part of foundation between 3 sensors 
Next, we present the data obtained with a monitoring system located on one building of the group under observation. This is a typical brick 3-storey building with a length of about $30 \mathrm{~m}$ and a width of about $12 \mathrm{~m}$. The hydro-levelling sensors are located at the characteristic points on the basement (Fig. 3a). Fig. 3b,c,d show the changes in deformation parameters recorded during 2 years of observation. These parameters are: vertical displacement at the point of sensor location relative to the displacement of sensor 5 , angular distortion of the foundation part between 2 sensors and the curvature of the foundation part between 3 sensors. Figs. 3c, d show that the relative settlements and angular distortions increase with time. However, the curvature varies insignificantly. By the end of the 2-year observation period, for this building the maximum settlement was $14 \mathrm{~mm}$, and the maximum rate of change of the relative settlement was up to $0.5 \mathrm{~mm}$ per month. Similar data were obtained for other buildings from the observed group.

Measurement of the surface slope using the HLS has an error, the value of which is determined by the measuring technique. Under ideal conditions, this approach introduces micron errors in the measurements of vertical displacements. However, for structures under real operating conditions (variable temperature gradients, height difference of HLS connecting tubes, oscillatory processes in the liquid caused by the operation of mechanisms), the error may increase by orders of magnitude reaching hundreds of microns. These errors inevitably must affect the obtained values of the inclination angles, which are calculated from vertical displacements.

The estimation of the influence of errors in the measurement of vertical displacements on the error of the inclination angles was carried out in the numerical experiment as follows. According to Fig. 3a, it is assumed that the HLS sensors are located on the foundation of the building at $N$ discrete points. We believe that the readings of each $i$-th sensor includes the determined value of the vertical displacements $U_{i}^{\text {true }}$ and the random error $\Delta U_{i}$

$$
U_{i}=U_{i}^{\text {true }}+\Delta U_{i}
$$

Then, the values of tilt angles $\varphi$ and direction angle $\alpha$ are represented in the following way:

$$
\begin{aligned}
& \varphi=\varphi^{\text {true }}+\Delta \varphi, \\
& \alpha=\alpha^{\text {true }}+\Delta \alpha,
\end{aligned}
$$

where $\varphi^{\text {true }}$ and $\alpha^{\text {true }}$ are calculated in terms of the values of $U_{i}^{\text {true }}$, and $\Delta \alpha$ and $\Delta \varphi$ are defined by the random quantities $\Delta U_{i}$.

In the numerical examples considered, the values of $U_{i}^{\text {true }}$ were prescribed such as to provide a tilt angle $\varphi^{\text {true }}=0.0001 \mathrm{rad}$ and three possible values of the direction angle: $\alpha^{\text {true }}=\left(0,45^{\circ}, 90^{\circ}\right)$. The deviation of a random value $\Delta U_{i}$ for each $i$-th sensor were set independently using a random number generator that ensures a uniform distribution of the probability density of this value in the interval from $-0.25 \mathrm{~mm}$ to $+0.25 \mathrm{~mm}$. This interval was chosen based on the experience of operating such systems. For $k$-realization of the set $\Delta U_{i}^{k}$, the values of $\Delta \varphi^{k}$ and $\Delta \alpha^{k}$ were determined for a given number of sensors $N$. For the specified number of sensors and the selected direction $\alpha^{\text {true }}, 900$ realizations of random deviations of vertical displacements were performed $(M=900)$. From the obtained statistical set, the following quantities are evaluated: 


$$
\begin{gathered}
\sigma_{\varphi}=\sqrt{\frac{\sum_{k=1}^{M}\left(\Delta \varphi^{k}-\overline{\Delta \varphi}\right)^{2}}{M-1}}, \sigma_{\alpha}=\sqrt{\frac{\sum_{k=1}^{M}\left(\Delta \alpha^{k}-\overline{\Delta \alpha}\right)^{2}}{M-1}}, \\
\varepsilon_{\varphi}=\frac{\Delta \varphi_{\max }-\Delta \varphi_{\min }}{2}, \quad \varepsilon_{\alpha}=\frac{\Delta \alpha_{\max }-\Delta \alpha_{\min }}{2} .
\end{gathered}
$$

where $\sigma_{\varphi}, \sigma_{\alpha}$ are the standard deviations and $\varepsilon_{\varphi}, \varepsilon_{\alpha}$ are the semi-range of the errors $\Delta \varphi$ and $\Delta \alpha ; \overline{\Delta \varphi}$ and $\overline{\Delta \alpha}$ are the sample means for these quantities [15].

The values of the standard deviation $\sigma_{\varphi}, \sigma_{\alpha}$ can be interpreted as the accuracy of estimating the angles $\varphi$ and $\alpha$ for random deviations in the readings of vertical displacement.

Numerical experiments were carried out for the buildings of three types, differing in the configuration of the foundations and in the length to width ratios (Tabl. 1)

Table 1. Building dimensions

\begin{tabular}{|c|c|c|c|}
\hline Building type & Length, $\mathrm{m}$ & Width, $\mathrm{m}$ & Numberof installed sensors \\
\hline I & 30 & 12 & 8 \\
\hline II & 60 & 12 & 6 \\
\hline III & 36 & 36 & $8-11$ \\
\hline
\end{tabular}

The experiments showed that the accuracy of determining the angles of inclination and orientation of the normal to the foundation plane depends on the number of sensors recording the vertical displacements. Fig. 4 shows the graphs of changes in, $\sigma_{\alpha}$ and $\sigma_{\varphi}$ depending on the number of sensors at three values of orientation angle $\alpha^{\text {true }}\left(0^{\circ}, 45^{\circ}, 90^{\circ}\right)$. The figures correspond to different types of buildings.

As can be seen from the figures, with increasing number of sensors, the range of random variables reduces to some values. Further increase in the number of sensors does not lead to a significant change in the magnitude of the range. This minimum number of sensors, $\mathrm{N}_{\text {opt }}$, varies for different types of buildings and is equal to $10 \div 12$ for buildings of type I, $14 \div 16$ for buildings of type II and $20 \div 24$ for buildings of type III.

Analysis of the graphs in Fig. 4 shows that the accuracy of estimating the angles of inclination and the orientation of the normal to the foundation plane depends on the configuration of the sensor system and its location relative to the orientation of the normal. For the buildings with the same characteristic dimensions in the plan (type III), the accuracy of angle estimation is practically independent of the orientation of the building. Elongated buildings with more sensors on their long side have the least deviation in the angles values if they are oriented in the direction of the normal inclination $(\alpha=0)$.

In general, it can be concluded that the registration of vertical displacements with an accuracy of $\pm 0.25 \mathrm{~mm}$ ensures the accuracy in determining the tilt angle of the normal $\Delta \varphi$ of $2 \cdot 10^{-6}-6 \cdot 10^{-6} \mathrm{rad}$ and the accuracy in determining the orientation angle of $1^{\circ}-3.5^{\circ}$.

The presented results allow us to estimate the error in measuring the orientation of the normal to the earth's surface within the building site caused by random perturbations of the readings of the HLS sensors. The obtained dependences make it possible to determine the optimal number of sensors for various types of buildings required to ensure the smallest measurement error. 


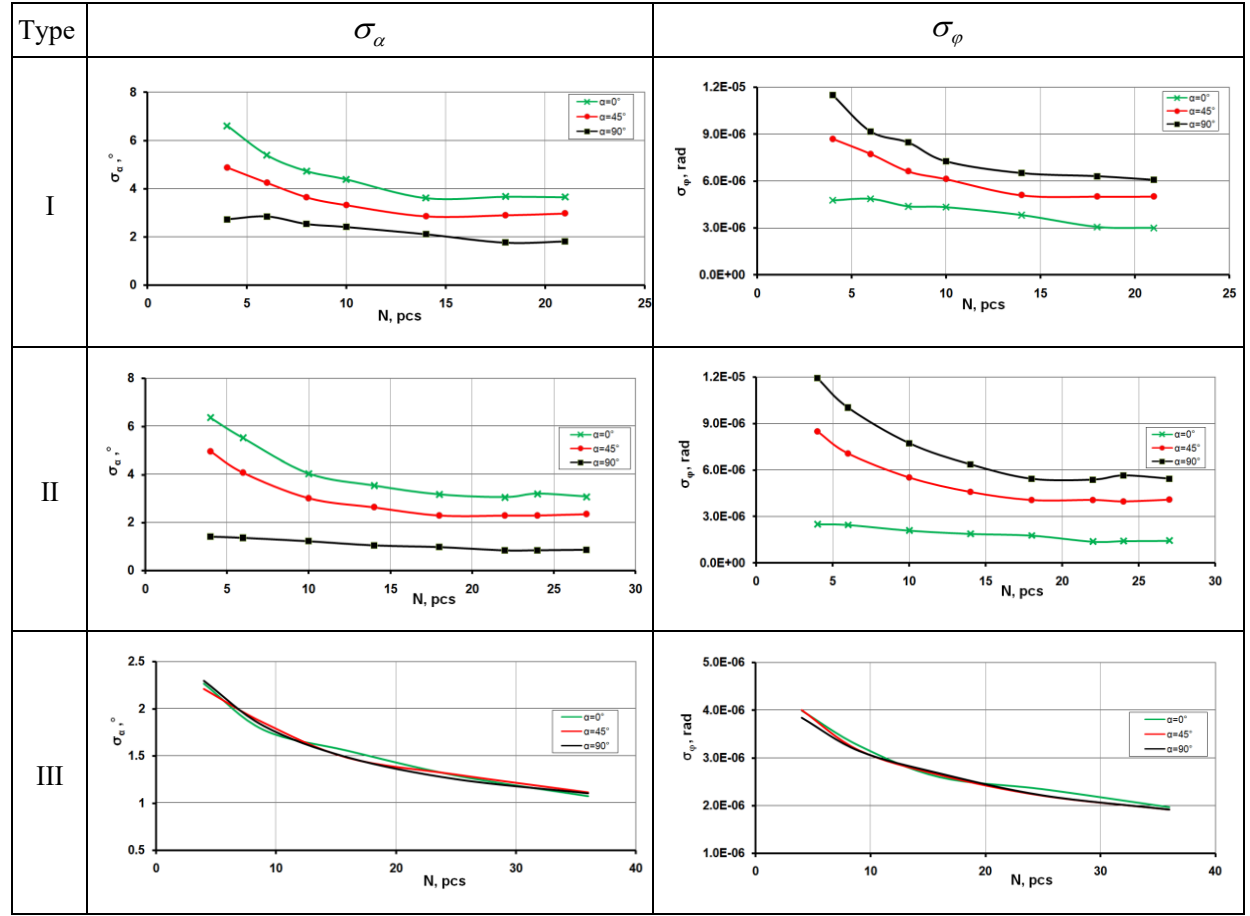

Fig. 4. Standard deviations $\sigma_{\alpha}$ and $\sigma_{\varphi}$ depending on the number of sensors for the building of different types

\section{The results of monitoring tilt angles of the rock massif}

The developed distributed monitoring system, installed on the foundations of 40 residential buildings, provided information on the slopes of the normal to the earth's surface on an area of $\sim 5 \mathrm{~km}^{2}$. The observation area is located in the city of Berezniki, Perm Region, in the area above the salt mines. A feature of this territory is the location of the mine field directly under the residential area. The processes occurring in the thickness of the mined salt rocks significantly affect the condition of buildings and structures. They led to the emergency condition of a number of buildings, and also caused the formation of sinkholes in the vicinity of the observed objects. Therefore, monitoring the deformation state of the earth's surface in this region is of considerable practical interest. It substantiates the approaches to predicting deformation processes in the soil massif, and allows one to estimate the period of safe operation of individual houses. We present the results of observations of the tilt angles in the monitoring site obtained during continuous monitoring over a number of years. Figure 6a shows a map of the area with a group of buildings. The HLS-based monitoring systems are installed at the foundations of the buildings, which are marked with numbers 18. The buildings differ in size and shape and are equipped with the settlement registration systems that include different numbers of sensors (Table 1). In Fig. 5a, each of the buildings is marked with an arrow, the direction of which coincides with the projection to the horizontal plane of the normal onto the foundation plane, and the length is proportional to the tilt of the normal. The figure demonstrates the state of the earth's surface, formed 3.5 years after the beginning of monitoring. Based on the analysis of the entire set of arrows (their direction and length), one can single out the localization region of the most intensive deformations in the rock massif within the examined area (the shaded zone). These results 
are in good agreement with the information on the location and configuration of existing salt mines $[11,12]$.

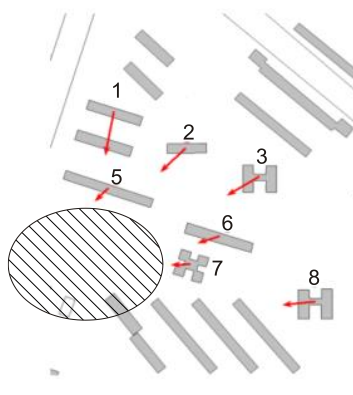

a
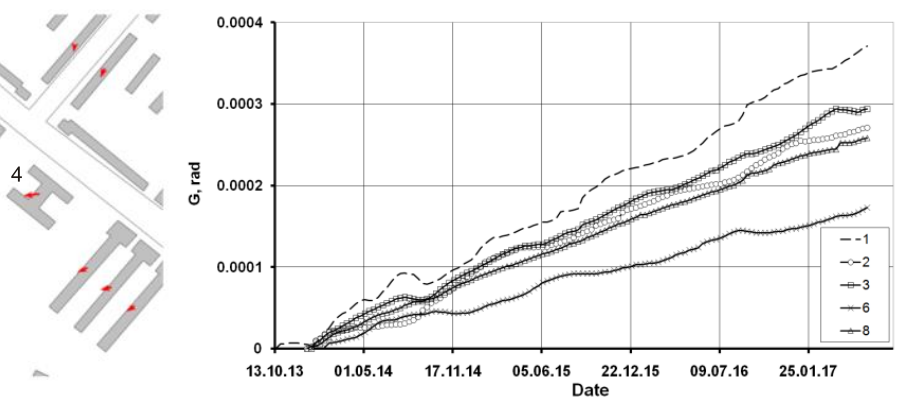

b

Fig. 5. Tilt angles of a group of buildings formed over a 3.5-year period (a) and their evolution (b)

Fig. $5 \mathrm{~b}$ illustrates the observed evolution of the tilt angles of the earth's surface at the locations of observed buildings (the curve numbers correspond to the building numbers in Fig. 5a). As is evident from the figure, over the observation period the greatest changes of about $0.35 \mathrm{rad}\left(0.02^{\circ}\right)$ in the tilt angle were recorded in the zone of location of house No. 1. The graphs show that the slope of the buildings changed in time with almost constant speed. The observed deviations from the linear dependence are due, in particular, to the errors of the HLS associated with seasonal changes in the temperature gradients inside buildings. The results of observation of the evolution of the rock surface shape accumulated for several years, allow us to conclude that the deformation processes are monotonous. Based on the obtained values of the average rates of changes in the tilt angles, we can predict their possible values in the interval of about several months.

\section{Conclusions}

An approach was proposed for monitoring a vast surface of the rock massif in a mining zone based on the analysis of the behavior of a set of buildings located in a given area. Each of the buildings is considered as a sensor of a distributed monitoring system, which records changes in the tilt angle of the normal to the surface as a result of distortion of the earth's surface. Measurements of the tilt angles of the building as a unit rigid body were based on the data of local automated monitoring systems, which measure settlements of the building foundations with HLS sensors. The applicability range of the proposed method was determined, the random error of the measurement method was estimated, and the optimal number of sensors for local monitoring systems was calculated.

Monitoring of the deformation state of a set of buildings for 3.5 years allowed us to reveal the localization of the zone of the most intensive deformation processes occurring deep in the rock massif. The obtained data not only allow assessing the deformation problems of the buildings equipped with monitoring system. They also help us to specify city areas potentially risky for maintenance of existing buildings and undesirable for construction of new buildings.

\section{Acknowledgements}

This research was performed under the grant of the Russian Science Foundation (grant № 14-29-00172). 


\section{References}

1. J.M. Pardo., A. Lozano, G. Herrera, J. Mulas, A. Rodriguez. Environmental Earth Sciences, 70:1957-1963 (2013)

2. P. Riley, P. Crowe. Coal 2006: Coal Operators' Conference: 266-275 (University of Wollongong and the Australasian Institute of Mining and Metallurgy, 2006)

3. T. Thongprapha, K. Fuenkajorn, J. Daemen. Tunnelling and Underground Space Technology, 46: 94-103 (2015)

4. Z.J. Zhang, C Wang, Y. Tang, Q.Y. Fu, H. Zhang. Int. J. Appl. Earth Observation and Geoinformation, 39(7):49-55 (2015)

5. H.D. Fan, D. Cheng, K.Z. Deng, B.Q. Chen, C.G. Zhu. Survey Review. 47(345):438445 (2015)

6. E. Can, S. Kuscu, C. Mekik. Int. J. of Coal Geology, 89: 62-69 (2012)

7. F. Mancini, F. Stecchi, F. Zanni, G. Gabbianelli. Environmental Geology. 58: 381-389 (2009)

8. H. Zhao, F. Ma, Y.Zhang, J.Guo. Environmental Earth Sciences, 68:1903-1911 (2013)

9. R. Tsvetkov, I. Shardakov, A. Shestakov, V. Yepin. Advances in Structural Integrity. Proceedings of SICE 2016: 129-136 (2018)

10. G. Ren, D.J. Reddish, B.N. Whittaker. Min. Sci. Technol. 5: 89-104 (1987)

11. A.A. Baryakh and N.A. Samodelkina. J. Min. Sci. 48 (6): 954-961 (2012)

12. A.A. Baryakh, S.Yu. Devyatkov, N.A. Samodelkina. Min. Sci. 52 (1): 36-45 (2016)

13. A.A. Baryakh and N.A. Samodelkina. J. Min. Sci. 4: 33-46 (2017)

14. I.N. Shardakov, A.P. Shestakov, R.V. Tsvetkov, V.V. Yepin. SSP. 243: 105-111 (2016)

15. D.R. Cox and D.V. Hinkley. Theoretical Statistics (Chapman and Hall, 1974) 